\title{
Design and construction of a new haptic interface
}

\section{Conference Paper}

Author(s):

Kunz, Andreas (D); Burri, Adrian

Publication date:

2000

Permanent link:

https://doi.org/10.3929/ethz-b-000082630

Rights / license:

In Copyright - Non-Commercial Use Permitted 
DETC2000/CIE-14582

\title{
DESIGN AND CONSTRUCTION OF A NEW HAPTIC INTERFACE
}

\author{
Andreas M. Kunz \\ Adrian Burri \\ Swiss Federal Institute of Technology Zurich \\ Center of Product Development \\ Tannenstrasse 3; CH-8092 Zurich; Switzerland \\ T: +41-1-6325771, F: +41-1-6321181, E: kunz@imes.mavt.ethz.ch
}

\section{ABSTRACT}

Virtual Reality becomes more and more important within the product development process. It enables the engineer to realize constraints or mistakes in the product design at a very early stage by viewing the digital geometric prototype. Beside viewing the design of a product, additional functionalities like simulation of assembling, the physically correct behavior of a machine or the machine control come into focus of interest. Therefore, the interaction modality of haptic feedback gains more and more importance for simulation tasks in virtual environments. However there are only a few portable haptic interfaces with which the user can experience in a natural way the sensation of force feedback.

The scope of this paper is to present a new passive haptic interface that is lightweight and easy to use. Furthermore it has no constraints in the workspace and applies high forces to the fingertips of the user by blocking the natural grasping.

Keywords: force-feedback, virtual reality, haptic, interaction

\section{INTRODUCTION}

Virtual Reality is superior to other forms of humancomputer interaction, because it provides a real-time environment integrating several new communication modalities. These include stereo graphics, surround sound, tactile feedback and even taste and smell in the future. By providing such a rich sensorial interaction, virtual reality makes the user feel immersed in the simulation or application he is running. As opposed to looking at the computer, the user feels surrounded by a synthetic world with which he can interact [2].
The realism of current simulations is impacted by the lack of a good physical simulation and its feedback to the human sense, which is an important information channel to the user. Nowadays, most of the virtual environments are with vision and sound but without any tactile output. The information requirements of many tasks in the product development process, which need dexterous manipulation and the sense of touch, such as remote control of a robot to locate and feel surface contours of objects, is not met without tactile feedback. It is an important information channel and undeniable for virtual reality in the future.

In order to provide sufficient realism, the simulation must include physical constraints such as object rigidity, mass and weight, friction, dynamics, surface characteristics (smoothness and temperature), etc. As indicated in Fig. 1, these requirements are very difficult to meet, even if the haptic function is reduced only to the grasping of an object. Adding physical characteristics to virtual objects requires both powerful computing hardware and specialized input/output-tools. These I/O-devices are worn by the user and provide tactile and/or force feedback in response to the simulation scenario.

Major improvements in feedback actuators, sensors and computing hardware must lead to miniaturization, less cumbersome haptic interface devices and to an increase in the user's safety and freedom of motion. These improvements must lead to more natural, realistic and useful simulations. The computer must fit to the human and not vice versa. Only a good interaction with the virtual object, that can be seen, will guarantee success in the product development process. This gives the motivation of developing a new force-feedback device that is easy to use and allows the grasping of virtual objects. 


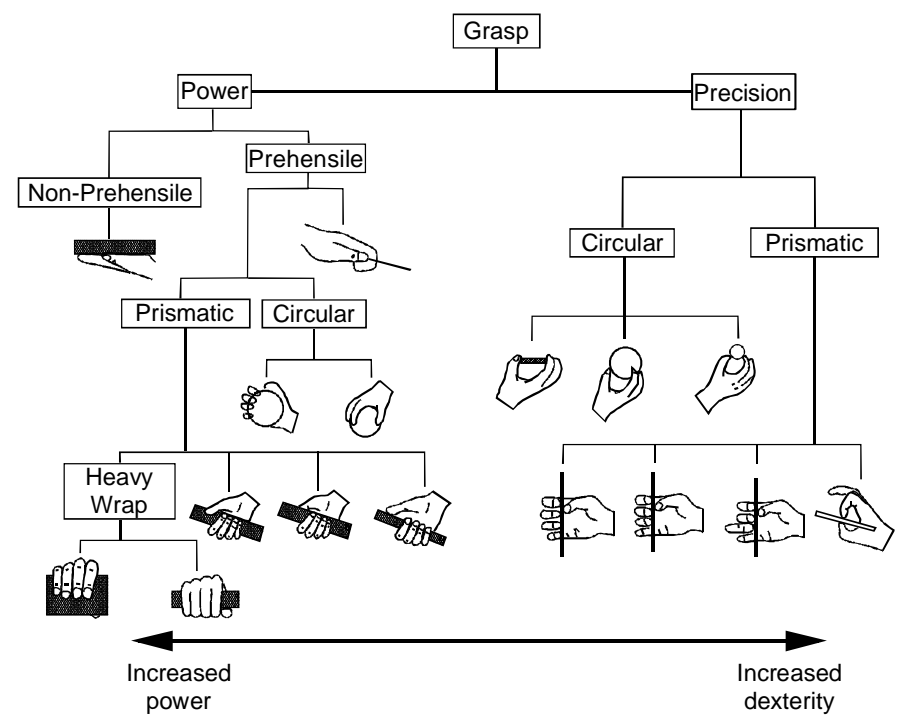

Fig. 1 Various hand grasp geometries [3]

The objective of this paper is to give an overview of the development of a new haptic interface (force-feedback device/FFD), with a simple position detection of the fingers and the possibility for a wireless communication with the computer.

\section{SPECIFICATION FOR A PORTABLE HAPTIC INTERFACE}

There are many haptic devices that have never left the status of a prototype and become a commercial product. In most cases, the design of the devices was the reason for this. They use electric motors, pneumatic cylinders or exoskeletons for generating and transporting the forces. Heavy constructions, which are only useable stationary, restrict the range of applications.

The advantage of nonportable interfaces lies in their ability to take the actuator weight away from the user. The disadvantage is a reduction in the user's freedom of motion, which makes the simulation less natural. In order to allow maximum freedom of motion to the user, it is necessary to use a portable force-feedback interface.

Existing force feedback devices had been evaluated and compared in order to define the specifications and requirements for a new haptic interface. Table 1 gives a comparison of some existing devices.
Tab.1 Comparison of some existing force feedback devices $[7 ; 8]$

\begin{tabular}{|l|c|c|c|c|c|}
\hline \multicolumn{1}{|c|}{ Interface } & Weight & Usability & Movability & $\begin{array}{c}\text { Applied } \\
\text { Force }\end{array}$ & $\begin{array}{c}\text { Controlled } \\
\text { Movement }\end{array}$ \\
\hline $\begin{array}{l}\text { Univ. of Tsukuba } \\
\text { Hand Masters }\end{array}$ & + & $+/-$ & + & $+/-$ & -- \\
\hline LRP Hand Master & $?$ & -- & + & - & ++ \\
\hline ARTS Hand FFD & $?$ & $+/-$ & + & -- & - \\
\hline $\begin{array}{l}\text { EXOS SAFIRE } \\
\text { Hand Master }\end{array}$ & -- & -- & - & $?$ & -- \\
\hline $\begin{array}{l}\text { Virtex CyberForce } \\
\text { Glove }\end{array}$ & + & + & + & + & + \\
\hline $\begin{array}{l}\text { Rutgers Master I } \\
\text { and II }\end{array}$ & ++ & - & -- & -- & - \\
\hline $\begin{array}{l}\text { IBM V-Flexor } \\
\text { Virtex } \\
\text { CyberGrasp }\end{array}$ & + & + & -- & $+/-$ & $+/-$ \\
\hline
\end{tabular}

For a portable haptic interface, the following general requirements are of concern:

- low weight

- low energy consumption

- simple design

- safe electrical and mechanical use

- comfort of use

- little calibration / no calibration

- high repetition frequency [5]

- low manufacturing costs (compared to existing devices)

A portable haptic interface denotes an actuating and/or a sensing structure, which is attached on the users body (back, chest, arm or palm). Such portable masters are more difficult to design because there are limitations in overall weight and volume, dictated by the need to avoid user fatigue during the use of the device.

As the new device is designed to be portable, cableconnections to the computer or to a power supply are no longer necessary. This implies the specification for low energy consumption (for battery use) for the whole device, especially for the actuators to produce the force for blocking the fingers. To realize a high comfort of use, it is recommended that the new device is easy to use and easy to fit to different users. Characteristics such as little or no calibration, and no training phase for the users or the computer are as important as electrical and mechanical safety. This means that the electrical specification of the device must not exceed international limits for electrical safety. Although there are no restrictions for the mechanical force, it is highly recommended that the actuators do not exceed human power to avoid injury in a case of malfunction. A high repetition frequency allows grasping with increased dexterity, i.e. for the recognition of a virtual object in complete darkness. 


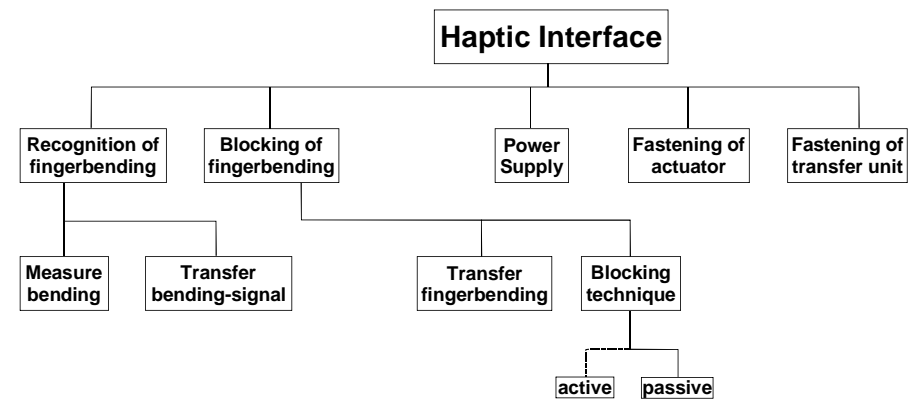

Fig. 2 Schematic diagram of a haptic interface

Fig. 2 shows the main subassemblies of a haptic interface, that have to be realized. In this paper the most important part, i.e. the blocking element, will be discussed in detail.

\section{FEASIBILITY STUDY AND PRELIMINARY ERGONOMIC INVESTIGATIONS}

The main task of a haptic interface is to produce a counteracting force to the human, when grasping a virtual object (Fig. 2). Therefore the interface needs an active element to produce this reacting force.

Interfaces can be separated into two groups depending on the way in which this force is produced:

- active force feedback devices

- passive force feedback devices

An active force feedback device requires actuators with the ability to produce a sufficient reacting force to the human muscles. This means that forces up to $50 \mathrm{~N}$ must be realized by the active elements (for heavy wrap), which leads to big and heavy actuators, such as motors or pneumatic cylinders. In general, active force-feedback devices have a high power consumption, because actuators must be supplied as long as the active force is needed.

The principle of a passive force-feedback device is blocking the closing movement (bending) of the fingers by the use of a shape intersection. In this case, the active element only has to activate a bolt, that produces the reacting force by the shape intersection. Very small active elements can be used, because they only have to move the bolt into a self locking position. The power consumption is very low because a supply is only needed in the moment of activation. The disadvantage of the shape intersection principle is that it is not possible to generate different stiffnesses of a virtual object. Every object feels as stiff as the material and the construction of the force feedback device itself.
Tab. 2 Comparison among active and passive force feedback devices

\begin{tabular}{|c|c|c|}
\hline & $\begin{array}{c}\text { Active } \\
\text { FFD }\end{array}$ & Passive FFD \\
\hline required force & $50 \mathrm{~N}$ & approx. 1 N \\
\hline Duration of force & during blocking & only for triggering \\
\hline Power consumption & high & low \\
\hline $\begin{array}{c}\text { Actuator size and } \\
\text { weight }\end{array}$ & high & low \\
\hline
\end{tabular}

To fulfill all of the above constraints, the decision was made to realize a passive FFD. For a first haptical sensation in a virtual environment, it is sufficient to feel the presence of an object as the main information on the haptical channel.

In a preliminary ergonomic investigation, it was determined how the length of a straight line on the back of the hand is lengthened when changing the hands shape from a flat hand to a fist [6] as shown in Fig. 3.
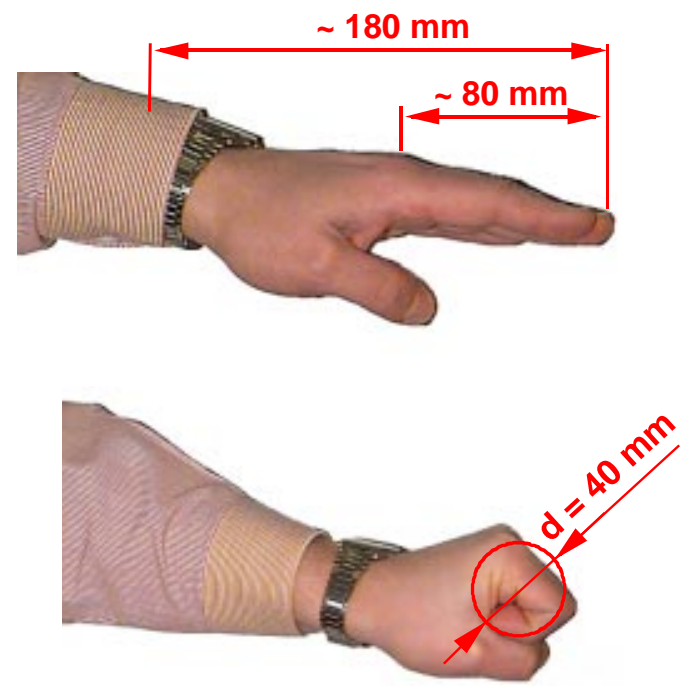

Fig. 3 Typical geometry of a hand

Assuming a straight line on the back of the hand with a total length of $180 \mathrm{~mm}$ and a length of $80 \mathrm{~mm}$ that is being bent when clenching a fist. If the fingers are completely round (for instance when grasping very small objects), the phalanx of the fingers is approximately a segment of a circle with $270^{\circ}$. Measuring the length between two marked points on the fingertip and nearby the wrist shows a lengthening of approximately $15 \mathrm{~mm}$, which can be used for detection and blocking of the fingers.

The ergonomic investigation also determined that the best place for placing the blocking elements is shortly beyond the wrist. In order to guarantee comfortable use, the maximum available space for all five blocking elements (referring to the 
five fingers) should not exceed a volume of 30(length)x50(width)x20(height) $\mathrm{mm}$.

\section{DESIGN AND CONSTRUCTION PROCESS}

The basic idea of the new haptic interface is to use this lengthening of a distance between the fingertip and the wrist to:

- detect the bending of the fingers

- block the linear movement and therefore the bending of the fingers

The general construction layout of the haptic interface is shown in Fig. 4. The objective of the interface is to restrain the closing motion of the hand. This implies that the motion of the fingers must be transferred to a Bowden-wire. The Bowdenwire itself is imbedded into a glove and connected to a fingertip attachment and to the lock element on the other side. It is important that the Bowden-wire is fixed exactly on the back of the hand and the fingers without the possibility to slip aside. This would cause a malfunction of force-feedback device (Fig.4).

In the case of a collision between the users hand (the bending fingers) and the virtual object (which is detected by the VR-software), this element locks the motion of the Bowdenwire and thus also the further bending of the fingers.

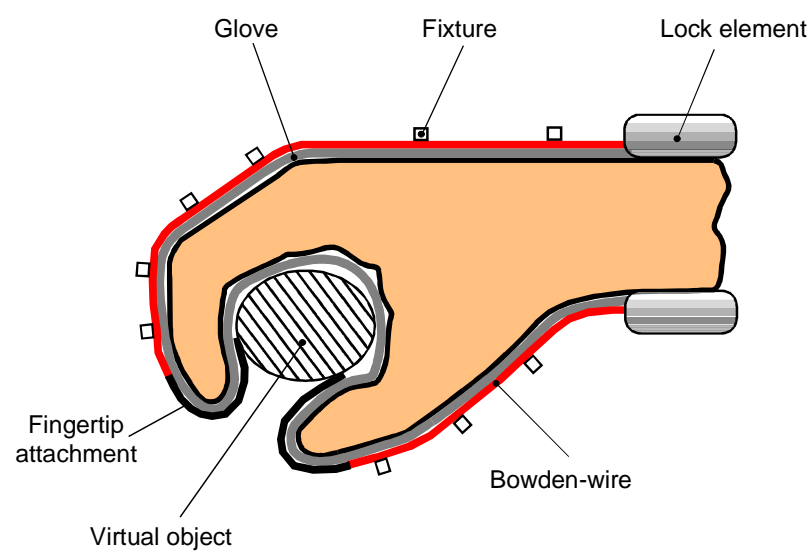

Fig. 4 General construction layout (longitudinal section)

The essential part of the force-feedback device is the lock element which is illustrated in a longitudinal section in Fig. 5

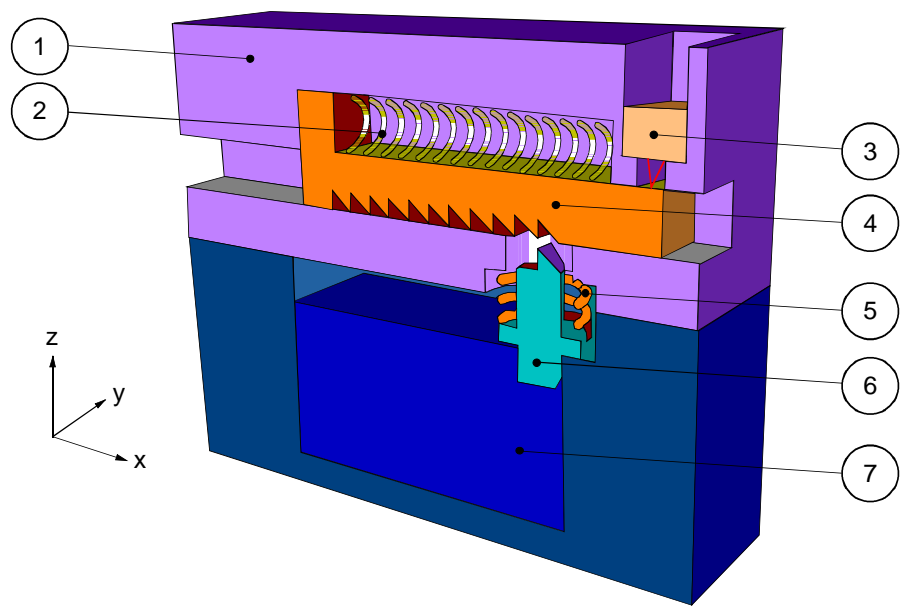

Fig. 5 Schematic layout of the blocking element (longitudinal section)

The bending of the fingers is transferred via a Bowden-wire to a linear movement of a toothed rack (4). The toothed rack will be moved in the $\mathrm{x}$-direction if the fingers are bent. In the opposite direction, the opening motion is supported by a spring (2). The movement of the toothed rack can be blocked by a bolt with a toothed head (6), which will be lifted by an electromagnet (7). In order to activate the blocking element, a short electric impulse is given tothe electromagnet and the lever is lifted. When the teeth engaged, an automatic locking occurs by the a closing shape and the power for the electromagnet can be switched off. A return spring (5) supports the reset of the locker when the virtual object is let go. Furthermore, the position of the toothed rack can be detected by a barcode on the back of the toothed rack, which is measured by a reflection light barrier (3). Counting the impulses that are generated by the reflection light barrier gives information about the position of the toothed rack and therefore about the bending of the fingers.

In the virtual environment, collision detection between the users hand and the virtual object is done by the software. In the test setup, the VD2-software by vr-com ${ }^{\odot}$ (a spin-off of the Fraunhofer institute Germany) was run. In case of a collision, an event signal is created and sent to the FFD via the serial port. The signals of the serial port are transmitted via a wireless datalink to the FFD. In this very first setup, only one output signal is provided for the blocking elements of all five fingers in order to save space, costs and weight. Only an electrical interface is provided between the blocking element and the serial port for an electrical isolation. The short electrical impulse, which is needed to activate the locking element allows it to use very small electromagnets. It is possible to realize 20 times the value of the nomimal wattage of an electromagnet if the duty cycle is short enough. The duty cycle is defined as:

$$
\text { Dutycycle }=\frac{\text { "ON" pulse time }}{\text { ON"+"OFF" pulse time }}
$$


The characteristic of the used electromagnet with a size of 20 (length) $x 15$ (width) $x$ 7(height) is shown in the Fig. 6 .

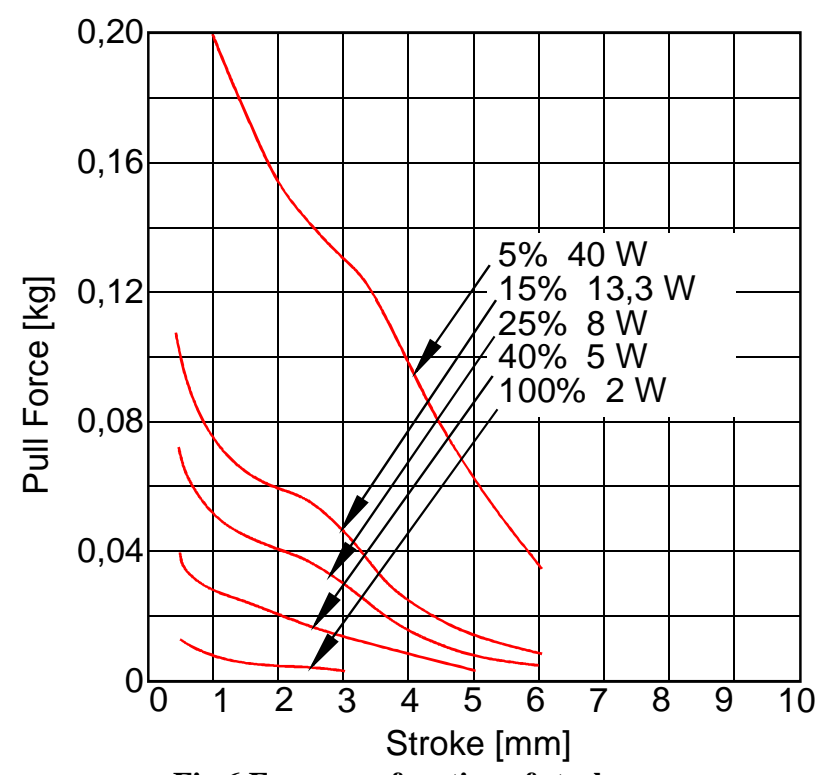

Fig.6 Force as a function of stroke

As a matter of fact the pull force depends on the gap between the end off the electromagnet and the ferromagnetic material. Using a solenoid the gap is represented by the stroke.

The most critical part of the locking element is the bolt itself and therefore it has been taken to an extra examination.

The bolt of the locking element is modeled as a cantilever beam as shown in Fig. 7:

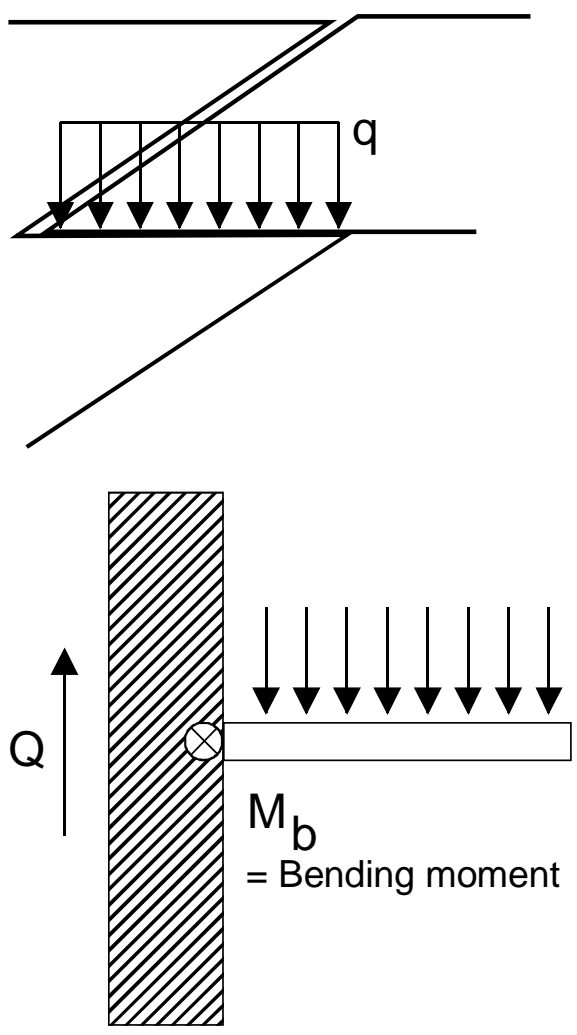

Fig. 7 Modeling of the tooth

The bolt is assumed to have a length and width of $1 \mathrm{~mm}$ each. In the case of blocking the contact pressure, torque and shear are the loads on this geometry. An external force of $\mathrm{F}=50$ $\mathrm{N}$ is assumed and the reference stress has been calculated. The result is a reference stress of $\sigma_{v}=266 \mathrm{~N} / \mathrm{mm}^{2}$ that implies no specific constraints to the material.

The action principle shown above must be slightly modified in order to fulfill the geometric constraint in height [4]. This change, as shown in Fig. 8 allows it to reduce the total height and furthermore to eliminate the readjusting spring for the locker bolt.

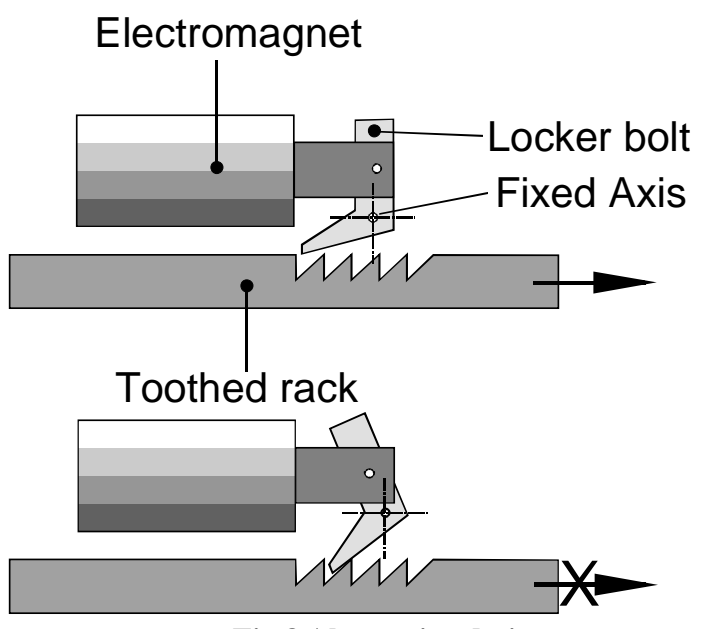

Fig.8 Alternative design 
The electrical components, i.e. the wireless datalink and the analysis of the electrical position signal, will be not be discussed here.

Figure 9 gives a small impression of the size of the very first prototype, realized in this case for only one finger:

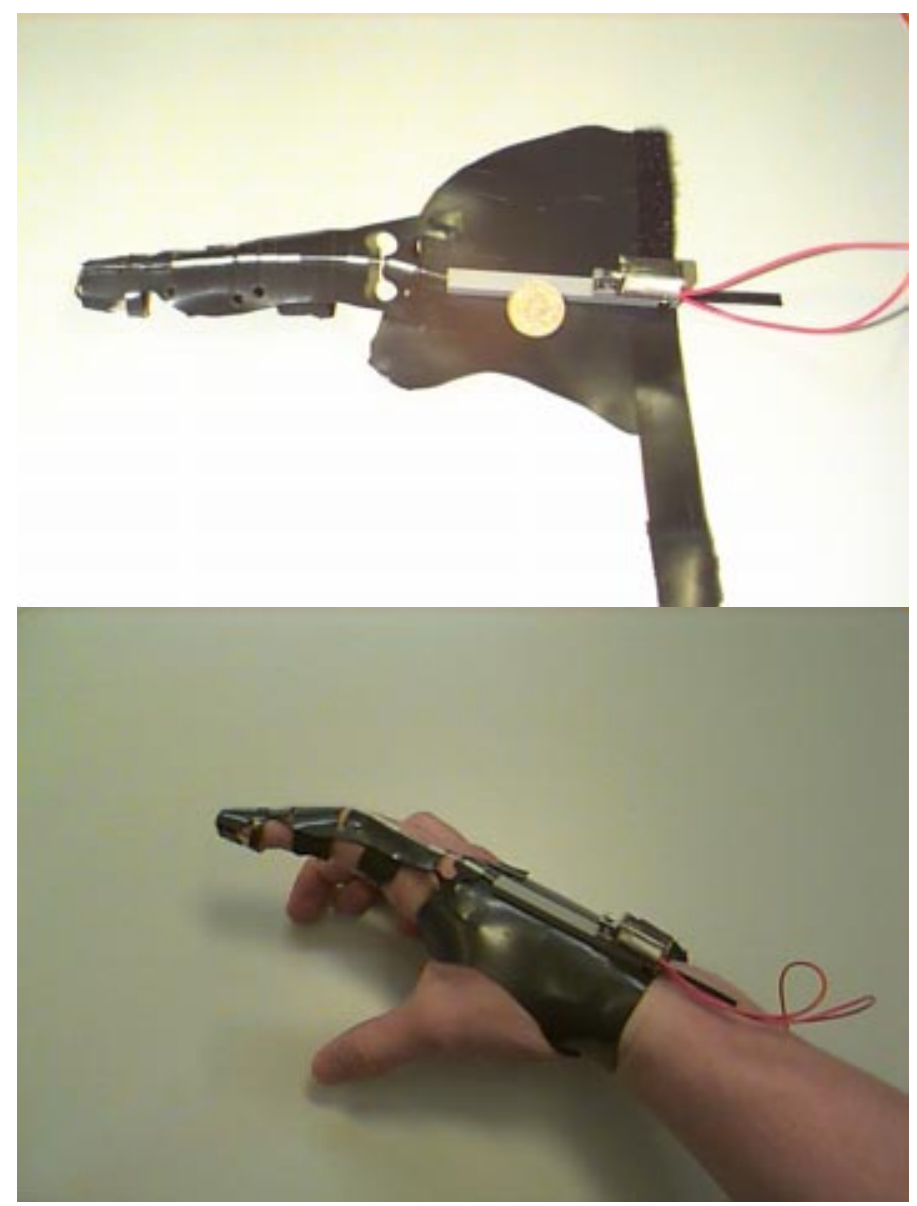

Fig. 9 First prototype for one finger

\section{FIRST EXPERIENCES}

The first grasping experiments with the new force feedback device demonstrated that it is possible to feel a good haptic sensation with this low cost solution. Neither the Bowden-wire on the back of the fingers, nor the attachment of the blocking element for the counteracting force irritates the haptic sensation

Another result of the first experiments was that it is not yet possible to feel the presence of a virtual object by touching it, because the activation of the actuators does not have any affect on the sensing fingertips when the fingers are not bent.

A generated counteracting force of approximately $20 \mathrm{~N}$ per finger (in the case of blocking) is sufficient to give a good haptic sensation. However, under full load there is a small immersive irritation at the wrist.

\section{FUTURE WORK}

While this work is a significant step forward in the development of force-feedback devices, it has its limitations. The next step in developing this force-feedback device will be to extend its functionality to all five fingers. Therefore the present design of the glove must be modified in order to avoid the immersive irritation at the wrist. Future work will focus on additional actuators to stimulate the sensing by the fingertips. Furthermore, sensing and grasping of an object will be combined in order to fulfill the needs of the product development process.

\section{SUMMARY AND CONCLUSIONS}

A new mechanical design of a portable haptic interface has been presented. With respect to the required forces, to the electrical safety and to a low power consumption, a self locking mechanism, which is activated by a relay, has been selected. It was taken into account to realize a cheap and easy to rebuilt solution. A first prototype for one finger was built and first experiences show a good haptic sensation.

Compared to the force feedback devices of Tab. 1, the new device provides a very low weight combined with a very good applicable force. The freedom of motion is also very good due to its wireless data link. The usability of the first prototype is bad and has to be improved. Depending on the concept only one movement (the fingerbending) can be controlled.

\section{REFERENCES}

1. W.J.Elspass; A.M.Kunz: „Portable haptic interface with active functional design"; Smart Structures and Materials; Proceeding of SPIE Vol. 3668; 1.-4. March 1999

2. G.C.Burdea; ,Force and touch feedback for virtual reality"; John Wiley \& Sons Inc. 1996; ISBN 0-471-02141-5

3. M.Cutkovsky, R.Howe; „Human grasp choice and robotic grasp analysis"; in S. Venkataram and T. Iberall Eds., Dextrous robot hands; Springer Verlag 1990; pp. 5-31

4. A.Burri; „Funktionale Gestaltung eines haptischen Interfaces"; Diploma thesis ETH Zurich 1999

5. K.Shimoga; „Finger Force and Touch Feedback in Dextrous Telemanipulation"; Proceedings of NASA-CIRSSE International Conference on Intelligent Systems of Space Exploration; NASA; Greebelt; MD; September 1992

6. A. Faller; „Der Körper des Menschen“; Deutscher Taschenbuch Verlag; 1998

7. Virtual Technologies Inc.; http://www.virtex.com/products/hw_products/cybergrasp.html

8. Rutgers State University of New Jersey; http://www.caip.rutgers.edu/vrlab/rm2.html 